\title{
Stellar Population Models
}

\author{
Claudia Maraston \\ University of Oxford, Denys Wilkinson Building, Keble Road, Oxford OX1 3RH, UK \\ email: maraston@astro.ox.ac.uk
}

\begin{abstract}
I review the current status of stellar population modelling, with particular emphasis on the treatment of the Thermally-Pulsing Asymptotic Giant Branch stellar phase. I discuss how much the physical properties of galaxies that are derived applying these models to real data, depend on the adopted model.
\end{abstract}

Keywords. stars: AGB and post-AGB, galaxies: evolution

\section{Introduction}

Stellar population models are the tool for interpreting the light of galaxies, specifically for obtaining the key properties such as ages, metallicities, masses. The latter in turn set meaningful constraints to galaxy formation models, since stellar evolution timescales are independent of cosmology. Obviously, the characteristics of the stellar population model play a central role in determining what are improperly referred to as observational properties. It is therefore crucial to check whether the models include all relevant phases of stellar evolution, and if they can reproduce the properties of simple systems like globular clusters, that, rather than galaxies, are actually the appropriate templates of stellar population models. In this review I shall focus on the Thermally-Pulsing Asymptotic Giant Branch (TP-AGB) phase of stellar evolution, that is the most discrepant ingredient of the various models and has however a strong impact on the properties of $\sim 1$ Gyr stellar populations, like those existing in high-redshift galaxies.

\section{TP-AGB phase in stellar population models}

The integrated properties of a synthetic stellar population are obtained by adding up the contributions by individual stars. A crucial issue for the correct evaluation of the total luminosity is the coverage of all relevant stellar evolutionary phases. In young stellar populations with ages smaller than $\lesssim 0.2 \mathrm{Gyr}$, most of the energy comes, besides the Main Sequence, from luminous Helium-burning stars, while in old stellar populations ( $\gtrsim 2$ Gyr) this role goes to the Red Giant Branch. In the narrow age range in between $(0.2 \lesssim t /$ Gyr $\lesssim 2)$, the most important post-Main Sequence phase is the AGB, more specifically the part of it called Thermally-Pulsing Asymptotic Giant Branch (TP-AGB). The evolution through the TP-AGB phase, however, cannot be predicted fully by stellar evolution because of strong (and unknown) mass loss affecting the phase, the efficiency of which requires to be calibrated with data.

To overcome these difficulties, in the models by Maraston $(1998 ; 2005$, hereafter M98, M05) the description of the TP-AGB is semi-empirical. This is based on the calibration of the TP-AGB fuel consumption with data of Magellanic Clouds globular clusters (GCs, by Frogel et al. 1990). The Magellanic Clouds are the only sites that contain GCs in the age range - between 0.2 and $2 \mathrm{Gyr}$ - in which the contribution of the TP-AGB is relevant (Frogel et al. 1990). Moreover, ages and metallicities of these GCs are independently 
known, which allows a calibration to be performed. Also the spectral energy distributions of individual TP-AGB stars are taken from observations (Lancon \& Mouhcine 2002, see M05 for full details). The TP-AGB phase is responsible of the largest discrepancies in the synthetic spectral energy distributions (SEDs) of different evolutionary synthesis codes (M05). Figure 1 depicts the SED of a 1 Gyr, solar metallicity single burst stellar
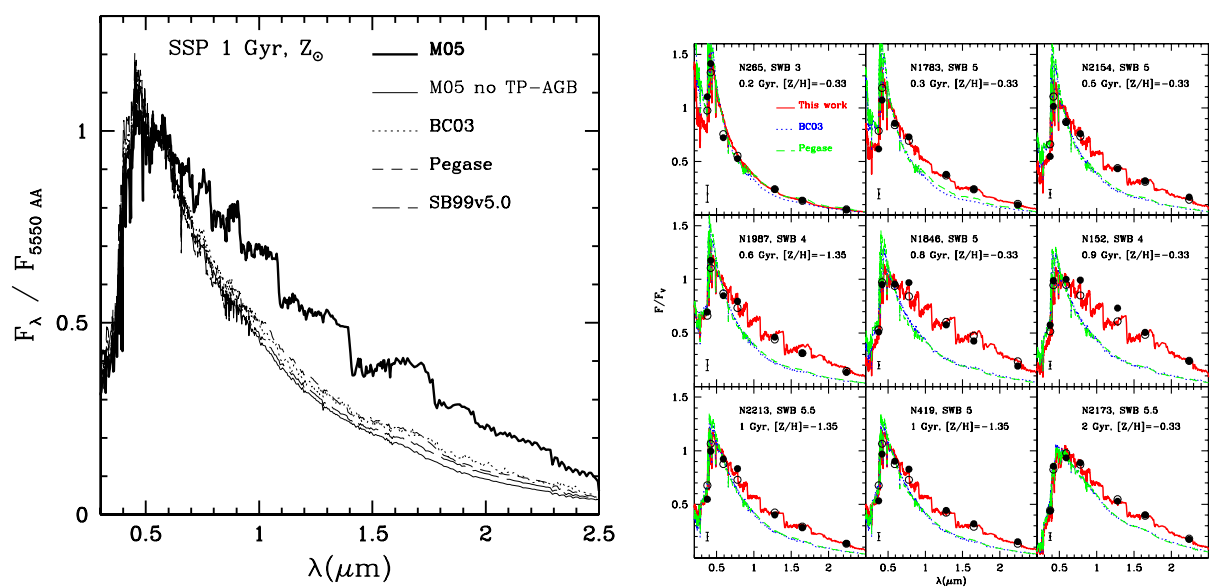

Figure 1. Left-hand panel. Synthetic SEDs of 1 Gyr single burst population with solar metallicity, according to different codes, M05, Bruzual \& Charlot (2003, BC03), Fioc \& Rocca-Volmerange (1997, Pegase), Vazques \& Leitherer (1999, SB99). The solid thin line shows a M05 model in which the TP-AGB phase was not included. Right-hand panel. Comparison of the M05 models (thick lines) with the observed photometric SEDs of LMC GCs (filled symbols), of which IDs, ages and metallicities are labelled. Other models from the literature are shown as thin lines. Both plots are adapted from M05.

population according to different codes. The M05 models stick out for having the highest amount of near-IR flux and for displaying the spectral molecular absorptions due to Carbon-rich and Oxygen-rich stars. These are the effects of the TP-AGB phase. As a consequence, the M05 models are redder and more luminous than others in the age-range where the TP-AGB is important, e.g. between 0.2 and 2 Gyr. The other models have a negligible contribution by the TP-AGB phase since the latter is not included fully in the Padova isochrones on which these models are based.

\section{Input stellar tracks}

Another source of uncertainty is the treatment of the convective overshooting in stellar tracks. Most evolutionary population synthesis codes adopt the Padova tracks, in which overshooting is included, while the M05 models use evolutionary computations in which overshooting is not included. The latter choice is motivated by the observational evidence that tracks with overshooting perform less well in matching the observed onset of the RGB phase as observed among the Magellanic Clouds GCs (Figure 2). This issue is not a secondary one, since it fixes the temporal scale of stellar population models, that ultimately determines galaxy ages. As can be seen in Figure 2, the overshooting as included in the Padova tracks delays the onset of the RGB until ages around 1 Gyr, with respect to non overshooting tracks in which the RGB develops at $t \sim 0.5$ Gyr. These differences impact on the derived mass of stellar systems (M05). 


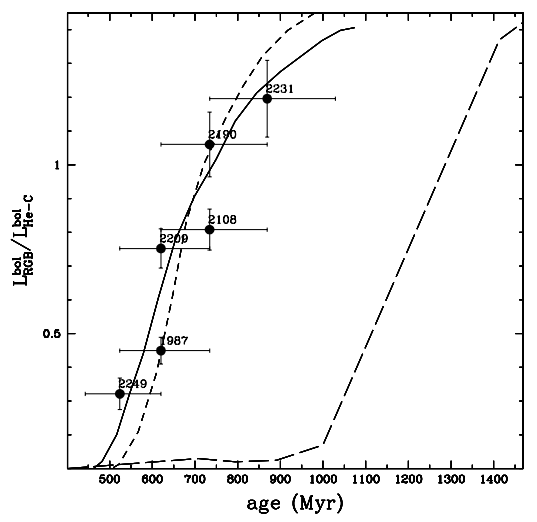

Figure 2. Onset of the RGB phase as measured by the luminosity ratio of RGB to HB stars in MC GCs. Stellar population models using the Padova (dashed lines, half-solar metallicity) and the Frascati tracks (solid and dotted lines, half-solar and 0.05-solar metallicity, respectivelly) are shown. From Ferraro et al. 2004.

\section{Effect on deriving ages and masses of (high-redshift) galaxies}

High-redshift galaxies must contain TP-AGB stars because the Universe at $z \sim 2-3$ is just 3-2 Gyr old, the right age range for the first generation of bright TP-AGB stars to be developed (Maraston 2004). This is also the age range in which the delayed onset of the RGB due to overshooting will have an effect on the predicted SED. As the rest-frame near-IR is now accessible up to very high-redshift thanks to the Spitzer Space Telescope, the different recipes for the red giants - TP-AGB and RGB - in the various stellar population models must have an effect on the derived properties of galaxies. Motivated by this idea, in Maraston et al. (2006) we analyze a sample of high-redshift galaxies from the Hubble Ultra Deep Field (HUDF) with spectroscopic redshifts and high-quality photometry including Spitzer data.

We find that indeed galaxy properties depend on the recipes for the TP-AGB (and RGB) phase in the models. Figure 3 shows, as an example, the SED fitting for two objects, for which some of the derived stellar properties - ages, metallicities and masses are labelled along with the spectroscopic redshifts. The analysis with the M05 models gives galaxies that are younger and less massive. The effect arises from the M05 SEDs becoming red at a younger age, because of the highest contribution by TP-AGB stars and the early onset of the RGB phase. In case of the BC03 models, older ages, those at which RGB stars are developed, are required to match the near-IR, and older ages imply higher masses because low-mass stars are less luminous. The effect on the stellar masses is shown in the right-hand panel of Figure 3. Note also that the quality of the data matching generally improves with the M05 models, as shown also by other groups (see van der Wel et al. 2006 and van der Wel, this volume).

\section{Conclusions}

I have discussed recent improvements in stellar population modelling, in particulor in the treatment of the TP-AGB phase of stellar evolution. The TP-AGB phase is the most important contributor to the energy of intermediate-age stellar populations (0.2- Gyr), producing half the bolometric luminosity and nearly all the one in the near-IR. These 

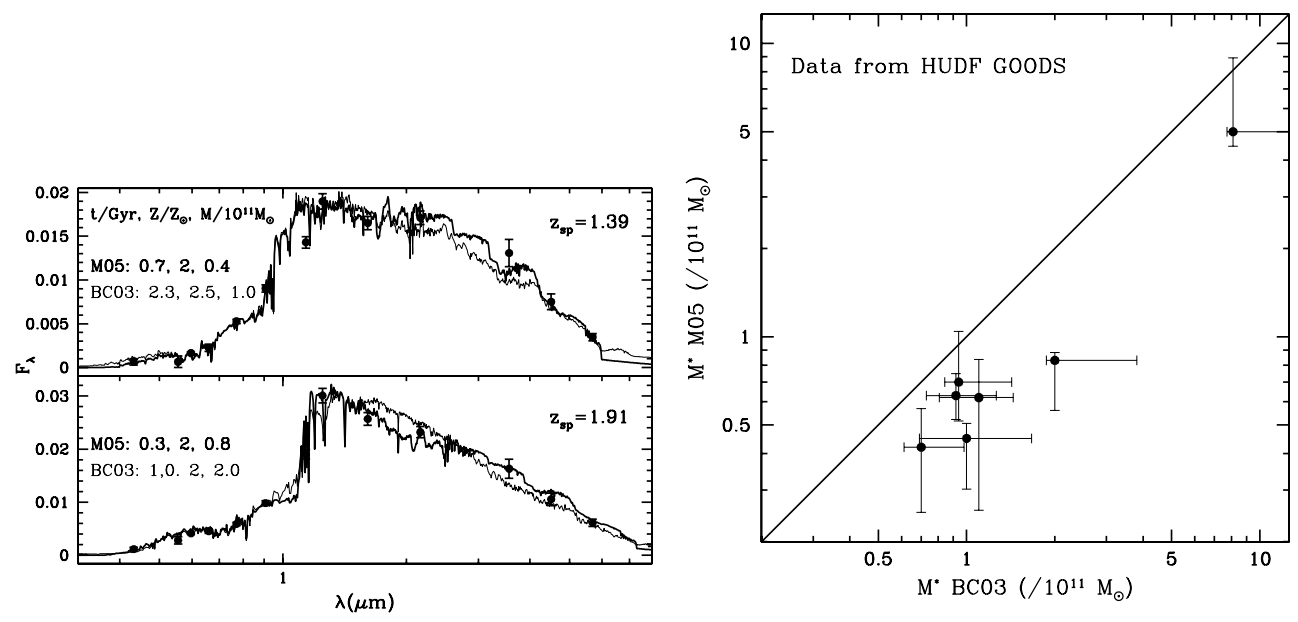

Figure 3. Left-hand panel. SED fitting for high-redshift galaxies from the HUDF. Results from the M05 and BC03 models are shown as solid and dotted lines, respectively. Ages, metallicities and stellar masses are labelled. Right-hand panel. Dependence of stellar masses on stellar population models.

models impact on the derivation of the properties of high-redshift galaxies, that, being young, are the ideal candidates to host at least some TP-AGB stars. I have also shown how the neglection of the TP-AGB phase in other models leads to the overestimation of galaxy masses and ages, which triggers misleading conclusions on galaxy formation.

\section{Acknowledgements}

I would like to thanks the organizers, Francoise Combes and Jan Paulos, for inviting me to an exciting and very informative symposium

\section{References}

Bruzual, G., \& Charlot, S. 1993, MNRAS, 344, 1000

Fioc, G., \& Rocca-Volmerange, B. 1997 (Pegase) MNRAS, 326, 950

Ferraro, F., et al. 2004, ApJ, 608, 772

Frogel, J.A., Mould, J., \& Blanco, V.M. 1990, ApJ, 352, 96

Lancón, A., \& Mouhcine, M. 2002

Maraston, C. 1998, ApJ, 300, 872

Maraston, C. 2004 in Multiwavelength mapping of galaxy formation and evolution, eds. A. Renzini \& R. Bender, Springer

Maraston, C. 2005, MNRAS, 362, 799

Maraston, C., et al. 2006, ApJ, in press, astro-ph/0604530

van der Wel, A. et al. 2006, ApJ, 636, L21

Vázques, G.A., \& Leitherer, C. SB99, 2005, ApJ, 621, 695

\section{Discussion}

Alice Shapley: You showed how the TP-AGB models differ from the BC03 models in the case of an SSP, then talked about different physical parameters you derive for high$\mathrm{z}$ galaxies. But SSPs are almost never used for $\mathrm{z}>2$ galaxies, that have independent estimates of young star formation. So what type of SF histories did you use to fit the high-z galaxies? 
Claudia Maraston: I use the SSPs to illustrate the basic differences between the unit models. But obviously in analysing galaxies, we use a wide range of SF histories from SSPs to constant star-formation, each for four chemical compositions, and with five options for the reddening by dust. This parameter space is much larger than what is usually considered in the literature, e.g. the sole solar metallicity combined with the Calzetti's slew (e.g. Shapley et al. 2005). 